\title{
Evolution of surface motor activation zones in hemiplegic patients during 20 sessions of FES therapy with multi-pad electrodes
}

\author{
Jovana Malešević (1, 2), Matija Štrbac (1, 3), Milica Isaković (1, 3), Vladimir Kojić (1, 4), \\ Ljubica Konstantinović (5, 6), Aleksandra Vidaković (5, 6), Suzana Dedijer (2, 6), Miloš \\ Kostić (7), Thierry Keller (7) \\ (1) Tecnalia Serbia Ltd., Belgrade, Serbia; (2) University of Belgrade, Belgrade, Serbia; (3) \\ University of Belgrade - School of Electrical Engineering, Belgrade, Serbia; (4) Innovation \\ Center, School of Electrical Engineering - University of Belgrade, Belgrade, Serbia; (5) \\ Faculty of Medicine - University of Belgrade, Serbia; (6) Clinic for Rehabilitation "Dr \\ Miroslav Zotović”, Belgrade, Serbia; (7) Tecnalia Research \& Innovation - Health Division, \\ San Sebastián, Spain.
}

This article is distributed under the terms of the Creative Commons Attribution Noncommercial License (CC BY-NC 4.0) which permits any noncommercial use, distribution, and reproduction in any medium, provided the original author(s) and source are credited.

\begin{abstract}
The purpose of this study was to examine surface motor activation zones for wrist, fingers and thumb extension movements and their temporal change during 20 therapy sessions using advanced multi-pad functional electrical stimulation system. Results from four hemiplegic patients indicate that certain zones have higher probability of eliciting each of the target movements. However, mutual overlap and variations of the zones are present not just between the subjects, but also on the intrasubject level, reflected through these session to session transformations of the selected virtual electrodes. The obtained results could be used as a priori knowledge for semi-automated optimization algorithm and could shorten the time required for calibration of the multi-pad electrode.
\end{abstract}

Key Words: Functional Electrical Stimulation, Multi-pad Electrode, Motor Activation Zones, Upper Extremity, Stroke

Eur J Transl Myol 2016; 26 (2): 175-180

Functional electrical stimulation (FES) system for grasping consists of an electrical stimulator and stimulation electrodes that serve as an interface for transmitting electrical pulses generated by the stimulator to the efferent nerves and motor points on the forearm that are in charge for hand opening and closing. ${ }^{1-3}$ These electrodes can be implanted, percutaneous or transcutaneous. The latter approach is the least invasive which makes it most attractive for motor control rehabilitation, but also imposes significant control issues. With transcutaneous electrical stimulation, the source of the electrical field that generates the depolarization of motor fibers is relatively distant from the target. To activate a target muscle, it is necessary to generate the appropriate voltage gradient in the vicinity of the neural pathway activating that muscle, which can be achieved by adjusting electrode position, current amplitude, pulse width and frequency. Eliciting functional movements, which proved to be the most beneficial rehabilitation method, ${ }^{3}$ requires synergic activation of multiple muscles. The main issue here is achieving selective stimulation, which has been the focus of many research groups. ${ }^{4-14}$ An effective method for accomplishing this is through multi-electrode stimulation, as described by. ${ }^{6-10,}{ }^{15}$ By adjusting the stimulation site and stimulation parameters for each electrode in the system, selective synergic muscle activation is possible; however, there is no general rule on the optimal stimulation site or parameters. Substantial differences in the anatomical layout of neural pathways among humans are the major issue. Therefore, the generation of a localized electrical fields needs to be set for each patient to ensure appropriate selective responses of the motor systems. Another problem, which is the focus of this paper, is the temporal change of these parameters attributed to factors such as patient's hydration, fatigue, spasticity, etc. These issues are arguably most pronounced in the case of grasp restauration, as the muscles that control finger extension often lie very close to wrist extension and eversion muscles. The usability of grasp restauration systems is highly correlated with the effort needed to position the electrodes and set up the stimulation parameters. A potentially effective method to improve 
FES therapy with multi-pad electrodes

Eur J Transl Myol 26 (2): 175-180

Table 1. Demographic and clinical data for patients who participated in the study. clinical data include baseline in action research arm test (arat) and fugl-meyer test ( $\mathrm{fm}$ ).

\begin{tabular}{|c|c|c|c|c|c|}
\hline Subject ID & Age & Months since onset & Affected side & ARAT & FM \\
\hline 1 & 50 & 2 & Left & 0 & 15 \\
\hline 2 & 45 & 1 & Right & 23 & 23 \\
\hline 3 & 62 & 3 & Left & 0 & 13 \\
\hline 4 & 55 & 40 & Left & 0 & 17 \\
\hline
\end{tabular}

the system usability is integration of electrodes in a garment which can be easily applied and removed from the patient's forearm. In our previous work we have addressed this issue by investigating general stimulation zones corresponding to mostly used motor responses necessary to create four basic grasps. ${ }^{16}$ There we proposed a design of a multi-pad electrode which could be easily set up to achieve a functional grasp. In this study we used the next generation of this electrode, with advanced, simpler design, which allows targeting of all stimulation zones of interest, even when fixed in an arbitrarily position. An advantage of such multi-pad electrode is the possibility to use virtual electrodes (VEs). A VE is a programmatically set group of pads positioned over a motor point, with a specific set of stimulation parameters, such that when activated together a specific movement is elicited. In the presented work the selectivity criteria was met when desired movements and designated VEs had one-to-one correspondence, i.e. when each defined virtual electrode elicited the designated movement, and no other movements. The focus of this paper is the temporal change of stimulation points, as well as intra and interpatient variability of stimulation zones. We observed stimulation positions for obtaining three important movements for spastic hand rehabilitation: wrist, fingers and thumb extension. Motor points for eliciting these three movements are usually intertwined, making selective stimulation a challenge. We present the results of a one month study on stroke survivors where changes in the stimulation locations were recorded in 20 consecutive sessions.

\section{Materials and Methods}

\section{A. Stimulation system}

For FES treatment of paretic hand we used advanced upper extremity rehabilitation system (Figure 1, top) from Tecnalia R\&I, San Sebastián, Spain (FESUpperExt). This system relies on INTFES V2 stimulator that enables time and space distributed stimulation over multi-pad electrode and can be controlled over Bluetooth by a custom designed host PC application. The application allows defining virtual electrodes (VEs) and storing them in the stimulator memory. A virtual electrode presents the sequence of asynchronous activation of one or more pads on the electrode with the appropriate stimulation intensities.
In this study we used a dedicated multi-pad electrode that can cover the motor points of interest for obtaining three important movements for spastic hand rehabilitation - wrist, fingers and thumb extension. The design of the electrode was ergonomic with the size and shape that can fit an average forearm. It consisted of 16 cathode pads in $4 \times 4$ configuration and one common anode designed to allow shifting and positioning over the wrist joint for different lengths of forearms (Figure 1, bottom). The stimulation frequency was set to $30 \mathrm{~Hz}$, pulse width to $250 \mu$ s and stimulation amplitude was set for each pad individually in the calibration process.

\section{B. Treatment protocol}

Each subject had 20 therapy sessions, 5 times per week for 4 weeks. Each session was divided into 3 sections: virtual electrode definition, muscle warm-up and exercise.

1) Virtual electrode definition

In virtual electrode definition section 3 VEs for the 3 desired hand movements were manually identified. Current intensity was set separately for each active pad within a VE. After initial system setup that includes positioning the electrode over the forearm, turning on the stimulator, starting the PC application and connecting the tablet PC with a control interface via Bluetooth, the process of current amplitude calibration was performed. In the first session stimulation parameters for all pads were set to $10 \mathrm{~mA}$ amplitude, $250 \mu \mathrm{s}$ pulse width and $30 \mathrm{~Hz}$ frequency. Then, for each VE, pads that belong to the adequate stimulation zones $^{16}$ were activated and if no motor response was identified by visual inspection, amplitude was increased by $1 \mathrm{~mA}$ until motor threshold was reached and the appropriate amplitude was identified or the patient reported unpleasant sensation. Pads with appropriate motor response were combined to produce a selective movement of interest. Number of pads was not limited, but the smallest number of pads was preferred. Pattern map containing 3 VEs with the optimized responses for wrist, fingers and thumb extension was saved to represent preferred VE configuration for that patient. This selection was stored in the stimulator memory as VE configuration for the muscle warm-up and exercise protocols. Location of the multi-pad electrode was marked on the forearm 


\section{FES therapy with multi-pad electrodes}

Eur J Transl Myol 26 (2): 175-180

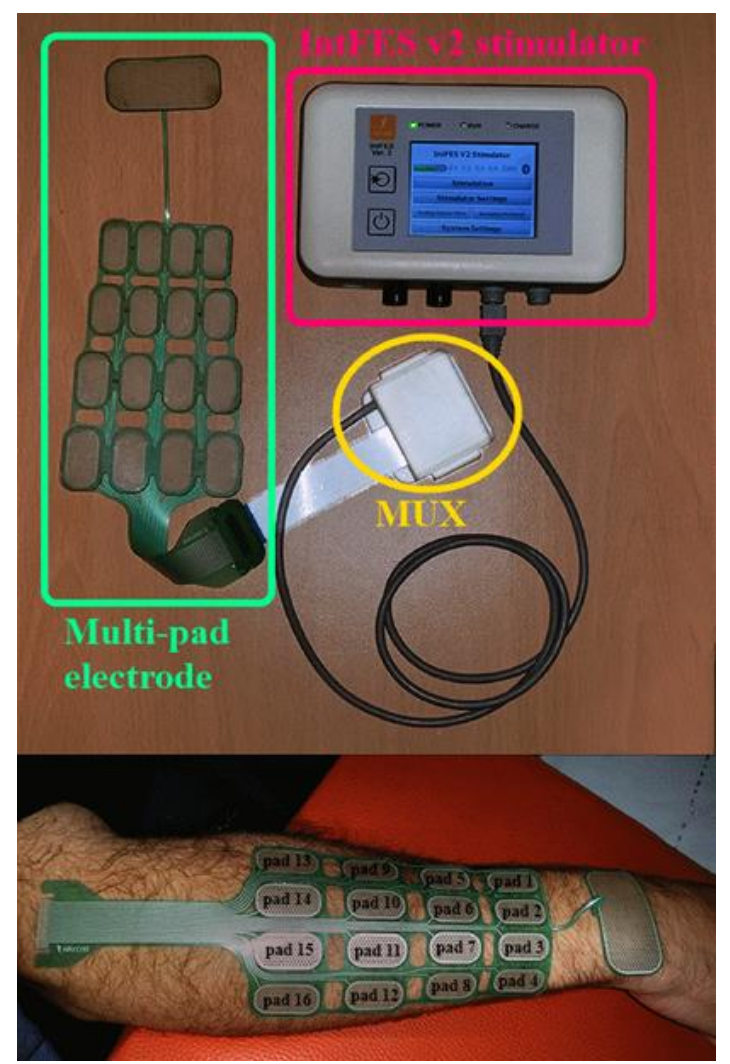

Fig 1. FESUpperExt system components (top) and the stimulation electrode on the forearm of one hemiplegic patient (bottom). The stimulation system consists of IntFES v2 stimulator (Tecnalia R\&I, San Sebastián, Spain) and the multi-pad electrode with 16 cathode pads designed to cover the motor points responsible for wrist, fingers and thumb extension.

after every session to ensure the same position of the electrode in the following session. Virtual electrode definition on each following day started from the pattern map used in the previous session. If VEs for the 3 defined movements didn't provide adequate response they were modified by manually adding/removing fields and changing amplitudes. In this manner same pads were favored and changes in VE configuration were discouraged.

2) Muscle warm-up

During the muscle warm-up protocol each pad that belonged to stimulation pattern map was individually activated for $2 \mathrm{~s}$. Pads were subsequently activated and pause between pads activation was $2 \mathrm{~s}$. Muscle warm-up lasted for $5 \mathrm{~min}$.

3) Exercise

Exercise section consisted of 4 different stimulation sequences that lasted for 5 minutes each:

1. $5 \mathrm{~s}$ of wrist extension with $5 \mathrm{~s}$ pause

2. $5 \mathrm{~s}$ of fingers extension with $5 \mathrm{~s}$ pause
3. $5 \mathrm{~s}$ of fingers and thumb extension with $5 \mathrm{~s}$ pause

4. $5 \mathrm{~s}$ of wrist, fingers and thumb extension with $5 \mathrm{~s}$ pause.

\section{Subjects}

Four male subjects (3 subacute and one chronic) with hemiplegia caused by iscemic stroke were included. Detailed demographic and clinical data for subjects is presented in Table I. All subjects received the conventional stroke rehabilitation program in addition to FES treatment explained above. The procedures and potential risks were explained to subjects individually and each signed a written consent. Ethical approval was obtained from the local ethics committee.

\section{Results}

Recorded pattern maps representing optimized VE configurations for wrist, finger and thumb extension movements were considered the output measure of the performed experiment. On Fig. 2 results for subjects 1 - 4 are presented. Daily, i.e. session to session, changes in VE configuration are presented in the upper panel. Here we present the evolution of electrode pad sets that comprised the VEs used for FES treatment of a specific patient through the course of therapy. The histogram of individual pad use is presented in the bottom panel of these figures. The number on each pad represents the number of therapy sessions when that pad was selected in the VE configuration. Maximal number of times that any pad could have been selected is 20 , which corresponds to the number of sessions that the therapy lasted for. The "/" sign denotes that the pad was never used in that VE. In this study both patients with the right and the left paretic hand took part. However, for easier interpretation, all electrode representations were remapped to the right hand electrode configuration, i.e. right side of the electrode drawing on bottom image is placed on the ulnar side of the forearm and narrow side is closer to the wrist. It should be noted that pad enumeration on this graphical representation of the electrode starts from the bottom right corner of the electrode, and moves through the electrode rows upwards, in order to match the electrode as presented the bottom panel of Figure 1.

\section{Discussion}

From the results shown in the lower panels for individual subjects of Fig. 2 we can notice that there are zones with high probability of eliciting one of the target movements, which is in accordance with our previous report. ${ }^{16}$ For each of the movements there are one or more highly recurring pads which, when observed in this way, may indicate the shape of the underlying structures, targeted by the stimulation. This zone is best defined in case of the wrist extension where pads 2 and/or 3 are present in VE definition in every instance. On the other hand, variability is largest for thumb extension, where in all but one patient such 
FES therapy with multi-pad electrodes

Eur J Transl Myol 26 (2): 175-180
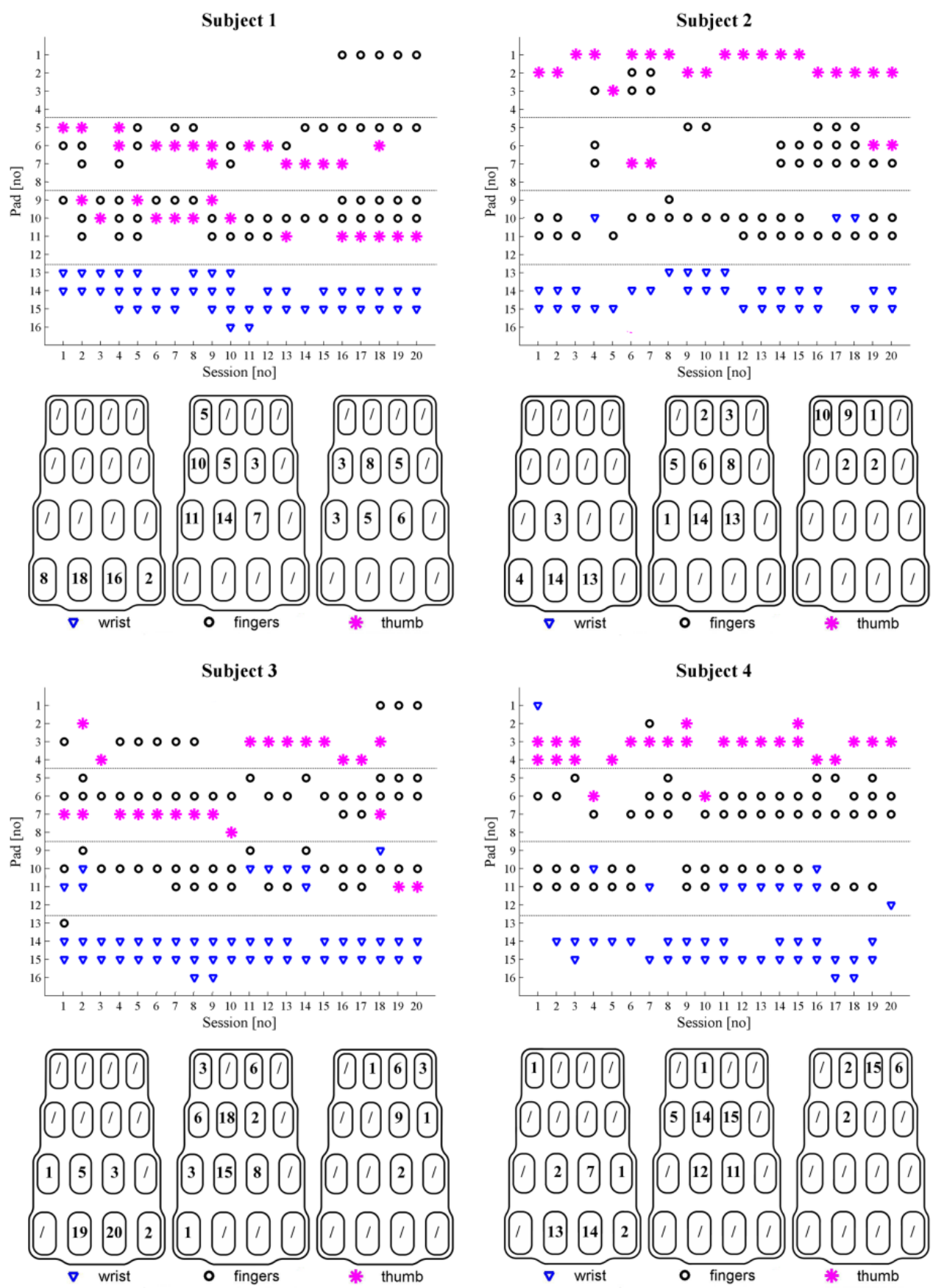

Fig 2. Virtual electrode configuration for subjects 1-4. The upper panel for each subject shows the evolution of the $V E$ configurations during 20 therapy sessions is presented. Therapy session of interest is presented on the horizontal and the selected pads for 3 VEs on the vertical axis. Pads included in wrist extension VE are marked with blue triangles, pads included in fingers extension VE are marked with black circles and pads included as thumb extension VE are marked with magenta asterisks. The bottom panel for each subject presents number of therapy sessions when each electrode pad was included in the VE for wrist, fingers and thumb extension is presented.

highly preferred pads weren't noted. In all three cases there are indications that there is a probability distribution which would fit any patient, but deriving such distribution requires much larger data sets. The temporal evolution of patterns, presented in top panels of
Fig.2 for each subject, reveals the truly stochastic nature of stimulation zones distribution. Even though pads used to elicit specific movements clearly cluster, there were many instances in which there was no overlap between two consecutive configurations. The 


\section{FES therapy with multi-pad electrodes}

Eur J Transl Myol 26 (2): 175-180

frequency of these occurrences was the greatest in the case of the thumb VE, and the lowest in the case of the wrist VE, which is in accordance with the spatial variability. On the other hand, there is no apparent trend in the change of the temporal variability amplitude, nor in the frequency of these changes. As the repeatability of electrodes placement was ensured by marked positions, these changes may be attributed to factors such as patient's hydration, fatigue, spasticity, changes in plasticity, etc.

A particularly interesting result, from the practical point of view, is the change of roles that occurs in some pads. This can be mostly observed in the pads comprising VEs for fingers and thumb extension, which is easily explained by the anatomic proximity of targeted motor points. However, this poses a major practical issue in the setup process. As there are several instances of significant change of VE configurations in consecutive sessions of each patient, e.g. complete inversion of finger and thumb VEs between day 9 and 10 for patient 1 , any configuration based on predetermined rules, which is not dynamically changed would often result in ineffective or even wrong VEs. Spatial clustering and indications of possibly identifiable probability distribution suggest that a VE configuration algorithm based on Bayesian decision theory can be developed in the future. Furthermore, the observed temporal variability implies the need for an adaptive algorithm with embedded intelligence of hand rehabilitation dynamics. Such a system could significantly simplify the setup process. However, as long as this system is operating in an open loop, in the end it will always rely on the operator's decisions and will be prone to mistakes due to the human factor. Closing the loop through sensors that can track hand and finger movements would increase the system complexity, but could significantly improve its performance.

\section{Contributions}

JM, MŠ, LjK, AV, MK and TK conceptualized the study. JM, MI, SD conducted the experiments. JM, MŠ and VK software and hardware design. JM analyzed the data. JM, MŠ and MK wrote the manuscript. All authors read and approved the final manuscript.

\section{Acknowledgement}

The authors would like to thank Andjelka Pjanović, Ivana Nikodijević and Milan Kecman, therapists from the Rehabilitation Clinic "Dr Miroslav Zotović" Belgrade, Serbia, for their expertise in work with the patients and the help they provided during this field study.

Research is supported by Tecnalia Research \& Innovation, Spain, by FIK, Spain, and by the Ministry of Education, Science and Technological Development of Serbia (Project no. 175016).

\section{Conflict of Interest}

The authors declare that there is no conflict of interest regarding the publication of this paper.

This paper was presented at the 20th IFESS Conference 'Hybrid approaches to FES', 8-10 June 2016, La Grande-Motte, France

\section{Corresponding Author}

Jovana Malešević is with Tecnalia Serbia Ltd., Belgrade, Serbia and University of Belgrade, Serbia, Vladetina 13, 11000 Belgrade, Serbia

E-mail: jovana.malesevic@tecnalia.com

E-mails of coAuthors

Matija Štrbac, matija.strbac@tecnalia.com

Milica Isaković, isakovic@etf.rs

Vladimir Kojić, koja@etf.rs

Ljubica Konstantinović, ljkonstantinovic@yahoo.com

Aleksandra Vidaković, aldragin@gmail.com

Suzana Dedijer, suzanadedijer@yahoo.com

Miloš Kostić, milos.kostic@tecnalia.com

Thierry Keller, thierry.keller@tecnalia.com

\section{References}

1. Popović MB, Popović DB, Tomović R. Control of arm movement: reaching synergies for neuroprosthesis with life-like control. J Aut Control 2002;12:9-15.

2. Hara Y, Ogawa S, Tsujiuchi K, Muraoka Y. A home-based rehabilitation program for the hemiplegic upper extremity by power-assisted functional electrical stimulation. Disabil Rehabil 2008;30:296-304.

3. Popović DB, Popović MB, Sinkjær $T$ et al. Therapy of paretic arm in hemiplegic subjects augmented with a neural prosthesis: a cross-over study. Can J Physiol Pharmacol 2004;82:749-56.

4. Sagi-Dolev AM, Prutchi D, Nathan RH. Threedimensional current density distribution under surface stimulation electrodes. Med Biol Eng Comput 1995;33:403-8.

5. Livshitz LM, Mizrahi J, Einziger PD. Interaction of array of finite electrodes with layered biological tissue: Effect of electrode size and configuration. IEEE Trans Neural Syst Rehabil Eng 2001;9:355-61.

6. Fujii T, Seki K, Handa Y. Development of a new FES system with trained super-multichannel surface electrodes. In: Proceedings of the 9th Annual Conference IFESS, 2004 Sept 6-9, Bournemouth, U.K., 2004. pp. 21-24.

7. Bijelić G, Popović-Bijelić A, Jorgovanović N et al. E actitrode: The new selective stimulation interface for functional movements in hemiplegics patients. Serbian J Elect Eng 2004;1:21-8.

8. Elsaify A, Fothergill JC, Peasgood W. A portable FES system incorporating an electrode array and feedback sensors. In: Proceedings of the 8th 


\section{FES therapy with multi-pad electrodes}

Eur J Transl Myol 26 (2): 175-180

International Workshop Functional Electrical Stimulation, Vienna, Austria, 2004. pp. 191-4.

9. Popović-Bijelić A, Bijelić G, Jorgovanović N et al. Multi-field surface electrode for selective electrical stimulation. Artif Organs 2005:29:448-52.

10. Kuhn A, Keller T, Micera S, Morari M. Array electrode design for transcutaneous electrical stimulation: A simulation study. Med Eng Phys 2009;31:945-51.

11. Elsaify A, Fothergill JC, Peasgood W. Portable FES systems optimizes electrode array using twitch response. In: Proceedings of the 9th Annual Conference IFESS, 2004 Sept 6-9, Bournemouth, U.K., 2004. pp. 27-9.

12. Keller T, Kuhn A. Electrodes for transcutaneous (surface) electrical stimulation. J Automat Contr 2008;18:35-45.
13. Lawrence M, Gross GP, Lang $M$ et al. Assessment of finger forces and wrist torques for functional grasp using new multichannel textile neuroprostheses. Artif Organs 2008;32:634-8, 2008.

14. Popović DB, Popović MB. Automatic determination of the optimal shape of a surface electrode: Selective stimulation. J Neurosci Methods 2009:178:174-81.

15. Livshitz LM, Mizrahi J, Einziger DP. Interaction of array of finite electrodes with layered biological tissue: Effect of electrode size and configuration. IEEE Trans Neural Syst Rehabil Eng 2001;9:355-61.

16. Popović-Maneski L, Kostić M, Bijelić G et al. Multi-pad electrode for effective grasping: design. IEEE Trans Neural Syst Rehabil Eng 2013;21:648-54. 